\title{
Note on Professor Naraniengar's Paper in the Proceedings of 1909-10.
}

\author{
By Professor F. Gomes Teixeira.
}

Permit me the liberty of presenting some remarks on an article by Professor Naraniengar inserted in Vol. XXVIII., p. 73, of the Proceedings of the Edinburgh Mathematical Society, 1909-1910.

In this Professor Naraniengar studies the locus of the points at which two sides of a triangle subtend equal or supplementary angles. Now, this curve is identical with the strophoid which was first considered by Barrow in Lectiones Geometricae (1669, p. 69). Van Rees has indeed shown in the Correspondance Mathématique de Quetelet (tome V., p. 361) that in a strophoid two chords having one of their extremities at the double point are able to be constructed in an infinite number of ways, so that they may subtend equal or supplementary angles at points on the curve; and M. Lebon has shown directly in the Journal de mathématiques speciales (1895) that the locus of points at which two sides of a triangle subtend equal or supplementary angles is a strophoid.

This remark does not lessen the merit of Professor Naraniengar's work, because one finds in it many propositions which enrich the theory of the curve mentioned, a curve which possesses remarkable properties, as can be seen in my Traité des courbes spéciales remarquables (tome I., p. 30).

One of these propositions is as follows:-The locus of the points of contact of the tangents (or the feet of the normals) drawn from a given point $A$ to the ellipses and hyperbolas having for real foci two given points $B$ and $C$ is identical with the curve at points on which $A B$ and $A C$ subtend equal or supplementary angles (that is to say, a strophoid). This curve passes through $\mathrm{A}, \mathrm{B}, \mathrm{C}$ and through the feet of the perpendiculars from $A$ on the axes of the confocal system, and has a node ut $A$. 
We add to this proposition of Professor Naraniengar's that this strophoid passes also through the imaginary foci of the conics. This is seen on putting $x= \pm i \delta$ in the equation found by the author :-

$$
\text { (i) }[x(x-h)+y(y-k)](h y-k x)=\delta^{2}(x-h)(y-k) \text {, }
$$

where $(h, k)$ are the coordinates of the point $\mathbf{A}$ referred to the axes of the conics, and $\delta$ is the distance of the real foci of the conies from the centre. This remark will soon be useful.

Applying the theorems of inversion to the results just obtained, we find a remarkable property of unicursal bicircular quartics.

If $A$ is taken as centre of inversion, the conics are transformed into unicursal bicircular quartics having their real double point at $A$, and having for foci the points corresponding to the foci of the conics. The cubic is transformed into an equilateral hyperbola passing through the real double point of the quartics, through the points corresponding to the real and imaginary foci, and through the points corresponding to the feet of the perpendiculars from $A$ on the axes. The axes are transformed into two circles, one of which passes through $A$ and the points corresponding to the real foci of the conics, the other passing through $A$ and the points corresponding to the imaginary foci of the conics. To the feet of the perpendiculars from $\mathbf{A}$ on the axes correspond the points of these circles diametrically opposite A.

We have then the following proposition:-The locus of the points of contact of tangents (or the feet of the normals) to the unicursal confocal bicircular quartics having the same real double point drawn from this double point is an equilateral hyperbola passing through this double point, through the foci of the quartics, and through the points diametrically opposite to this double point in the circles which pass through this point, and respectively through the two real foci and the two imaginary foci of the quartics.

If in particular the point $A$ is situated on one of the axes of symmetry of the conics, the bicircular quartics have evidently an axis of symmetry. But in this case the equation (i) shows that the cubic which it represents is reduced to the axis through $A$ and a circle through the two real foci or the two imaginary foci. Now this circle is transformed into a straight line through the two foci of the quartics corresponding to those of the conic through which 
the circle passes. Then, the locus of the points of contact of the tangents (and of the feet of the normals) to the unicursal bicircular quartics having the same axis of symmetry, the same foci, and the same double point, drawn from this double point are two straight lines, of which one passes through the real foci and the other through the imaginary foci.

[I knew that Professor Naraniengar's problem and many of his resulta could not claim to be now, but the treatment seemed to me fresh, and I thought his paper would be of interest to many of the readers of the Proceedings. For the history of the problem I refer, in addition to Professor Teixeirs's notes, to Loria's Spezielle algebraische und transcendente ebene Kurven. Theorie und Aeschichte (Bd. I., pp. 59-69), second edition.

Professor Teixeira's book, which he has kindly sent me, contains a very full disoussion of the ourve, with historical notes.

The property given by Professor Naraniengar in $\$ 20$ regarding confocals, and mentioned in the note above, is, of course, well known. I refer to Reye's Geometrie der. Lage (fourth edition), pp. 176-179, and Appendix 88, for beautiful easy proofs by projective geometry.

\section{J. Milurg, Co-editor of Proceedings.]}

